\title{
Exoplanet detection yield of a space-based Bracewell interferometer from small to medium satellites
}

\author{
Colin Dandumont, ${ }^{\mathrm{a} * *}$ Denis Defrère, ${ }^{\mathrm{a}}$ Jens Kammerer, ${ }^{\mathrm{b}, \mathrm{c}}$ Olivier Absil, ${ }^{\mathrm{d}}$ \\ Sascha P. Quanz, ${ }^{\mathrm{e}}$ and Jérôme Loicq ${ }^{\mathrm{a}}$ \\ ${ }^{a}$ Université de Liège, Centre Spatial de Liège, Liège, Belgium \\ ${ }^{b}$ European Southern Observatory, Garching, Germany \\ ${ }^{c}$ Australian National University, Research School of Astronomy and Astrophysics, Canberra, \\ Australian Capital Territory, Australia \\ ${ }^{\mathrm{d}}$ University of Liège, Space Sciences Technologies, Astrophysics Research Institute, \\ Liège, Belgium \\ ${ }^{\mathrm{e} E T H}$ Zurich, Institute for Particle Physics and Astrophysics, Zurich, Switzerland
}

\begin{abstract}
Space-based nulling interferometry is one of the most promising solutions to spectrally characterize the atmosphere of rocky exoplanets in the mid-infrared (3 to $20 \mu \mathrm{m})$. It provides both high angular resolution and starlight mitigation. This observing capability depends on several technologies. A CubeSat (up to $20 \mathrm{~kg}$ ) or a medium satellite (up to a few hundreds of $\mathrm{kg}$ ), using a Bracewell architecture on a single spacecraft could be an adequate technological precursor to a larger, flagship mission. Beyond technical challenges, the scientific return of such a small-scale mission needs to be assessed. We explore the exoplanet science cases for various missions (several satellite configurations and sizes). Based on physical parameters (diameter and wavelength) and thanks to a state-of-the-art planet population synthesis tool, the performance and the possible exoplanet detection yield of these configurations are presented. Without considering platform stability constraints, a CubeSat (baseline of $b \simeq 1 \mathrm{~m}$ and pupils diameter of $D \simeq 0.1 \mathrm{~m})$ could detect $\simeq 7$ Jovian exoplanets, a small satellite $(b \simeq 5 \mathrm{~m} / D \simeq 0.25 \mathrm{~m}) \simeq 120$ exoplanets, whereas a medium satellite $(b \simeq 12.5 \mathrm{~m} / D \simeq 0.5 \mathrm{~m})$ could detect $\sim 250$ exoplanets including 51 rocky planets within 20 pc. To complete our study, an analysis of the platform stability constraints (tip/tilt and optical path difference) is performed. Exoplanet studies impose very stringent requirements on both tip/tilt and OPD control. (C) 2020 Society of Photo-Optical Instrumentation Engineers (SPIE) [DOI: 10.1117/1.JATIS.6.3.035004]
\end{abstract}

Keywords: interferometry; satellites; exoplanets; astronomy; space optics.

Paper 20025 received Mar. 13, 2020; accepted for publication Aug. 13, 2020; published online Sep. 23, 2020.

\section{Introduction}

In less than 25 years since the first exoplanet discovery, ${ }^{1}$ more than 4000 exoplanets have been confirmed and more than 5000 are still awaiting confirmation. ${ }^{2}$ Most of these exoplanets have been detected by the transit and the radial velocity methods (resp. $\approx 75 \%$ and $20 \%$ of all confirmed planets). With these thousands of confirmed exoplanets and statistics derived from the Kepler satellite data, it has been shown that exoplanets should likely exist around each main-sequence star in our galaxy. ${ }^{3}$ The next big step is to spectrally characterize a larger number of rocky planets in the habitable zone of their star to analyze their atmosphere and search for biosignatures.

Characterizing exoplanet atmospheres is very challenging due to the high angular resolution needed and huge star/planet contrast. Nulling interferometry is one of the most promising direct methods to tackle this challenge in the visible, near-IR ( 0.5 to $3 \mu \mathrm{m}$ ) and mid-IR (3 to $20 \mu \mathrm{m}$ ) domains. All of these spectral bands contain several molecular absorption features (e.g., $\mathrm{H}_{2} \mathrm{O}$, $\mathrm{CO}_{2}, \mathrm{O}_{3}$, and $\left.\mathrm{CH}_{4}\right){ }^{4}$ The orbital parameters can be derived whatever the spectral band. Depending on the waveband, one can measure the planet temperature (mid-IR) or measure the planet radius (mid-IR) or a combination of the radius and the albedo (visible-NIR). ${ }^{5}$

*Address all correspondence to Colin Dandumont, E-mail: colin.dandumont@uliege.be

$2329-4124 / 2020 / \$ 28.00$ CC 2020 SPIE

J. Astron. Telesc. Instrum. Syst.

035004-1

Jul-Sep 2020 • Vol. 6(3) 
Table 1 Four studied configurations considered in this study from nano- to medium-size satellites.

\begin{tabular}{lcccc}
\hline \hline & CubeSat 6U & CubeSat 12U & PROBA-size & FKSI-concept \\
\hline Size & $0.6 \times 0.1 \times 0.1 \mathrm{~m}$ & $1.1 \times 0.1 \times 0.1 \mathrm{~m}$ & $1 \mathrm{~m}^{3}$ & \\
Baseline length $(\mathrm{m})$ & 0.5 & 1 & 5 & 12.5 \\
Pupil diameter $(\mathrm{m})$ & 0.08 & 0.08 & 0.25 & 0.5 \\
Optical train temperature $(\mathrm{K})$ & 150 & 150 & 100 & 60 \\
\hline \hline
\end{tabular}

The optical train temperature is discussed in Sec. 5.3. The tip/tilt angle and the RMS OPD for each configuration are, respectively, introduced in Secs. 4.1 and 4.2.

In 1978, Bracewell ${ }^{6}$ proposed a space-based interferometer with two apertures and a recombination of light in phase opposition. It produces a dark fringe on the line-of-sight and the stellar emission is strongly suppressed. For an off-axis source, the light can be transmitted depending on the baseline length and the wavelength, which define the interferometer transmission map. Even though the nulling interferometry principle was demonstrated on the ground on different telescopes ${ }^{7-9}$ a space-based mission is needed to avoid atmospheric effects (turbulence and opacity at specific wavelengths).$^{10}$ European Space Agency (ESA) and NASA studied several space-based missions during the 2000s [Darwin, Terrestrial Planet Finder-Interferometry (TPF-I), and FourierKelvin Stellar Interferometer (FKSI)] but none of them have ever flown. ${ }^{11}$ Research is, however, still active with high-altitude experiments such as Balloon Experimental Twin Telescope for Infrared Interferometry, a double-Fourier-Michelson interferometer placed on high-altitude scientific balloon which flown in 2017, ${ }^{12}$ or Planetary Imaging Concept Testbed Using a Rocket Experiment, a sounding rocket with a visible nulling coronagraph which flown in 2015 . $^{13,14}$ With the results from these missions, the current technology, future prospects, ${ }^{10}$ and the results from Kepler, an interferometric space-based mission can now be re-evaluated. The Large Interferometer For Exoplanets (LIFE) mission project is part of this re-evaluation. The goal of this space mission is to characterize the atmosphere of dozens of terrestrial planets at the midinfrared wavelengths and assess their potential habitability. ${ }^{15}$

This paper is dedicated to the analysis of the scientific return of a smaller mission. As nulling interferometry was never achieved in space, a smaller space-based mission can be considered as a useful prerequisite to demonstrate some key components before developing an L-class mission, such as LIFE. In this paper, four types of mission sizes (Table 1) are considered, all in a Bracewell configuration, e.g., with only two apertures with a recombination in the pupil plane. They are chosen to cover a large variety of spacecraft sizes, from nano- to medium-class satellites. Thanks to the planet population synthesis tool (P-POP) developed by Kammerer and Quanz, ${ }^{16}$ synthetic planet populations, based on the Kepler satellite data, are generated around a sample of 326 real nearby main-sequence stars (distance $<20 \mathrm{pc}$ ). From the analysis of signals and major noise sources (Sec. 3), a signal-to-noise ratio (SNR) is derived for a given exoplanet and a specific instrument (Sec. 4). With a Monte-Carlo approach, the expected detection yield is computed from 100 trials or universes leading to the generation of $\sim 86,000$ synthetic exoplanets. ${ }^{16}$

\section{Mission Architecture}

This section is dedicated to a short description of a fiber-coupled Bracewell interferometer. Figure 1 represents a simplified block diagram of such a configuration. The elements in the optical path are as follows.

- Two telescopes to collect the light from the star and the putative planet. They are mounted on the same structure in our case, spaced by a distance $b$ (baseline length).

- Tip/tilt mirrors to correct any tip/tilt errors in the beam. This correction is done thanks to fast steering mirrors. 
Dandumont et al.: Exoplanet detection yield of a space-based Bracewell interferometer...

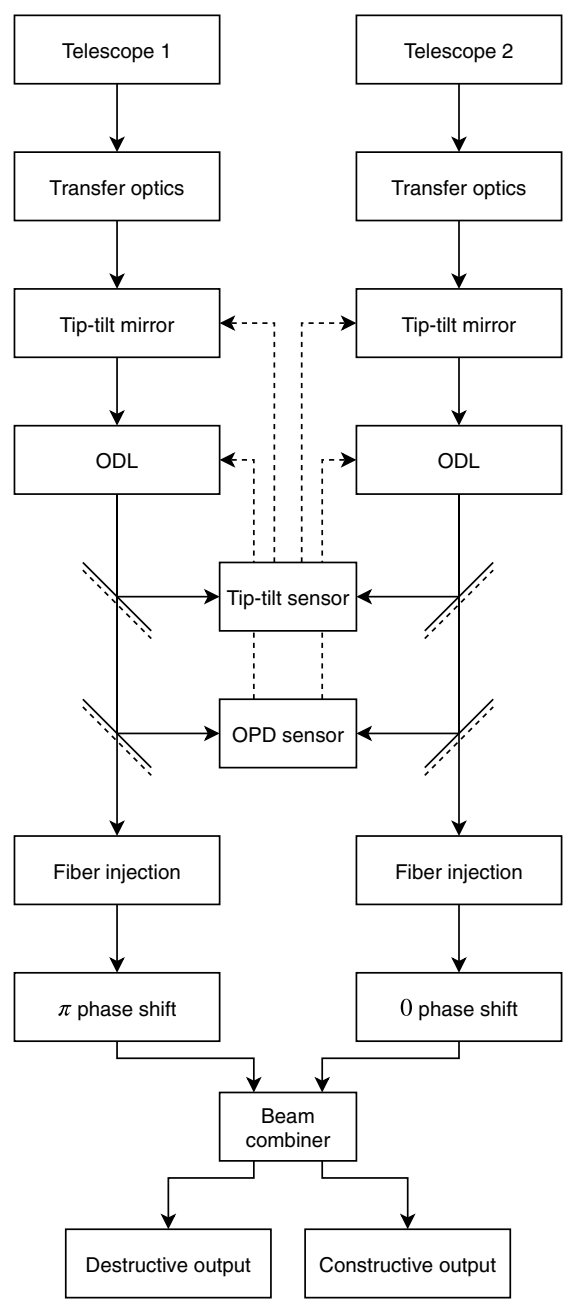

Fig. 1 Block diagram of a fibered Bracewell interferometer optical layout (simplified version of the one shown in Ref. 17).

- Optical delay lines (ODL) to correct any optical path differences (OPD).

- Tip/tilt sensors and OPD sensors to measure the corrections that need to be applied.

- A fiber injection system, after the OPD and tip/tilt corrections. These optical fibers (singlemode waveguides) are used as modal filtering. To avoid any chromatic effect, these two fibers must have the same length.

- An achromatic $\pi$ phase shift introduced in one arm of the interferometer to create the phase opposition in the line-of-sight of the interferometer and suppress the starlight.

- The beam combiner to receive the light from both apertures and create interference in the pupil plane.

- Two photodiodes: one at the constructive output and one at the destructive output.

In this study, four spacecraft configurations are considered (Table 1). They cover a variety of sizes: two CubeSats, a small satellite (PROBA) and, a medium satellite (FKSI). For each of them, a pupil diameter and a baseline length are chosen. The choice of the optical chain temperature is discussed in Sec. 5 as well as the optical path delay correction (ODL).

\subsection{CubeSat}

More than 1000 CubeSats have been launched so far and 3000 are expected to be launched in the six following years. ${ }^{18}$ They are part of the "NewSpace" trend, and thanks to the miniaturization 
of all platform components, it becomes reasonable to think about astronomical CubeSats. We are aware of at least two already launched CubeSats dedicated to transiting exoplanets: PicSat from the High Angular Resolution Astronomy group at the Paris Observatory/LESIA laboratory in France $^{19,20}$ and Arcsecond Space Telescope Enabling Research in Astrophysics from the Jet Propulsion Laboratory. ${ }^{21}$ The latter is the first CubeSat to have detected an exoplanet (superEarth 55 Cancri e). ${ }^{22}$

CubeSats are defined by their number of units. One unit, or $1 \mathrm{U}$, corresponds to $0.1 \times 0.1 \times$ $0.1 \mathrm{~m}$. In this paper, we consider two CubeSat concepts, a $6 \mathrm{U}$ and a 12U. 6U CubeSats are officially defined as $2 \times 3 \times 1 \mathrm{U}^{23}$ but an alternative exists. Although rarely used, nanoracks, who deploys CubeSats from the International Space Station, offers the possibility to launch a $6 \mathrm{U}$ CubeSat with a linear form factor $(6 \times 1 \times 1 \mathrm{U}){ }^{24}$ This choice will increase the baseline length of the satellite and so the angular resolution of the interferometer. For the $12 \mathrm{U}$ CubeSat, the idea is the same. Unfortunately, to our knowledge, no deployer for 12U CubeSats with a linear form factor exists. Therefore, we considered a typical $12 \mathrm{U}$ CubeSat $(2 \times 3 \times 2 \mathrm{U})$ with a deployable mechanism to obtain a $12 \times 1 \mathrm{U}$ CubeSat. In these configurations, both CubeSats have a maximized baseline length. Due to size constraints, the size of their primary mirrors is limited to $8 \mathrm{~cm}$.

\subsection{PROBA-Size}

The PROBA-size satellite is based on the PROBA family (Project for On-Board Autonomy) from the ESA. It is a satellite class designed around innovation at a reduced cost. Three successful missions are currently flying (PROBA-1 since 2001, PROBA-2 since 2009, and PROBA-V since 2013). The next one, PROBA-3 is planned to be launched soon. Composed of two spacecraft, these satellites will maintain formation autonomously to submillimeter precision in the lateral plane and millimeter precision in the longitudinal plane at distances of $150 \mathrm{~m}$ or more. ${ }^{25}$ It is an important step toward a large interferometric mission since formation flying is necessary to increase the baseline length up to a few hundred meters. ${ }^{10}$ The size of all PROBA satellites is around $1 \mathrm{~m}^{3}$. With deployable arms, one can assume to achieve a baseline length up to $5 \mathrm{~m}$.

\subsection{FKSI-Concept}

The FKSI-concept is derived from the nominal FKSI envisaged and developed by the NASA's Goddard Spaceflight Center. ${ }^{26}$ The goal was to have a medium mission before the TPF-I mission from NASA and to demonstrate some key technologies. Some studies have been done, but this mission was not further funded. ${ }^{26,27}$

\section{Determination of Signals and Noises}

This section presents a radiometric budget (signals and noise sources) of the instrument. All relevant signals, whether they are useful signals (planets) or external contributions (e.g., local zodiacal disk), are briefly introduced. Then the instrumental throughput is discussed as well as all considered noise sources. Figure 2 represents major photon sources in a schematic way. It shows all signals taken into account. To illustrate the radiometric budget calculated on a large hypothetical population of exoplanets, a randomly synthetic candidate is chosen from P-POP (see Sec. 5.1 for more information). Information about this hypothetical planet are available in Table 2 .

\subsection{Fluxes}

Figure 3 represents all the incoming signals (Jy) to the interferometer between 0.1 and $100 \mu \mathrm{m}$ (optical train temperature $T_{\text {optical }}$ at $150 \mathrm{~K}$ and $D=1 \mathrm{~m}$ ) for the randomly selected planet (Table 2).

All relevant signals are as follows.

- Stellar and Planetary Fluxes. Stars and planets are assumed to emit as black bodies. The full exoplanet spectral density flux is the sum of two contributions: the reflected flux, 


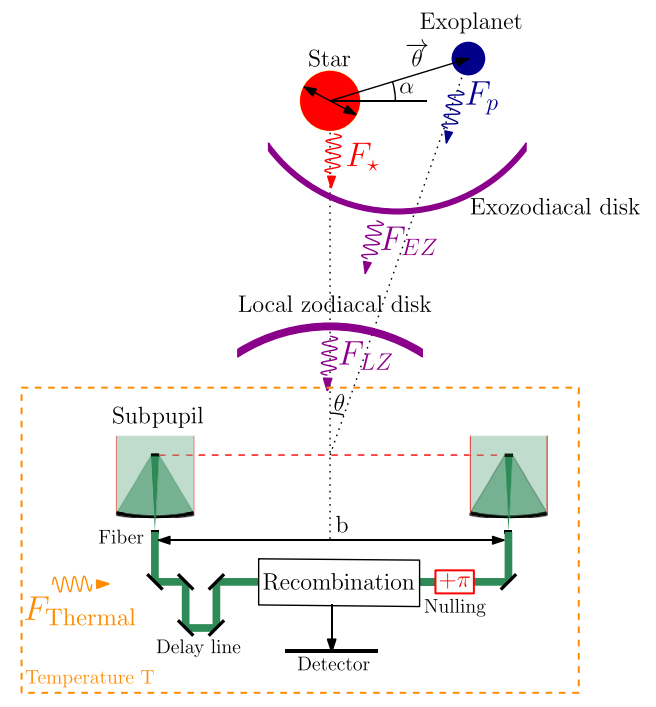

Fig. 2 Schematic presentation of a Bracewell nulling interferometer, represented with the main noise sources used in our radiometric budget. $F_{*}$, the stellar flux; $F_{p}$, the planetary flux; $F_{E Z}$, the exozodiacal flux; $F_{L Z}$, the zodiacal flux; and $F_{\text {thermal }}$, the thermal flux of the instrument (temperature, $T$ ).

Table 2 Parameters of a randomly selected synthetic exoplanet around Proxima Centauri. This planet is used as an example in the following figures.

\begin{tabular}{lclc}
\hline \hline Star & \multicolumn{2}{c}{ Putative planet } \\
\hline Star name & Proxima Centauri & Radius & $2.36 \mathrm{R}_{\oplus}$ \\
Spectral type & $\mathrm{M}$ & Period & 23 days \\
Radius & $0.14 \mathrm{R}_{\odot}$ & Semimajor axis & $0.077 \mathrm{AU}$ \\
Temperature & $3054 \mathrm{~K}$ & Maximum angular separation & 59 mas \\
Distance & $1.29 \mathrm{pc}$ & Geometric albedo & 0.5 \\
Exozodi level & 3.7 & Equilibrium temperature & $137 \mathrm{~K}$ \\
\hline \hline
\end{tabular}

which is a fraction of the emitted flux of the host star, and the thermal flux, which is directly related to the equilibrium temperature of the planet. ${ }^{28}$ The exoplanet curve (red dotted line) in Fig. 3 shows this double contribution. The planet position is considered here at its quadrature, which implicitly assumes that the planet is known in advance. If the orbital position is randomized, a fraction of the planets will no longer be detectable because they become too close angularly to the star. This effect was introduced by Brown in 2004 and is known as obscurational completeness. ${ }^{29}$ To increase the detection yield of a mission, revisits are therefore necessary. In our simulations, we consider only one visit per star. The impact of randomizing orbital positions on the detection yield is discussed in more detail in the conclusion.

- Local Zodiacal Emission. Local zodiacal dust is located between the orbit of Mercury and Jupiter. It scatters sunlight at every wavelength while it emits mainly in the mid-IR (thermal radiation). We assume that this dust has a temperature of $300 \mathrm{~K}$ in the habitable zone of the solar system. ${ }^{30}$ It adds an unwanted signal and can constrain the operating wavelength for the interferometer. It dominates any mid-IR emission in the sky. ${ }^{31}$ Thanks to the COBE mission, ${ }^{31}$ a model of the local zodiacal dust emission was derived and used by the Darwin team for a radiometric budget. ${ }^{28}$ The same model is used in this paper. The zodiacal disk 




Fig. 3 Input spectral flux density [ $\mathrm{Jy}]$ (reflection and thermal emission) of a synthetic planet (cf., Table 2) around Proxima Centauri compared to other sources (star, local zodiacal disk, exozodiacal disk, and thermal emission). The background flux is the sum of the contributions from the local zodiacal disk, the exozodiacal disk, and the thermal emission. Parameters: $D=1 \mathrm{~m}$ and $T_{\text {optical }}=150 \mathrm{~K}$.

has almost a symmetrical peak in the ecliptic. ${ }^{30}$ Therefore, the position of the observed stars relative to the interferometer is important and is taken into account through the ecliptic latitude of the potential planetary system generated by P-POP. The solar longitude is set to $\pi$ to have an anti-Sun direction and decrease the total emission. For a real mission, the satellite can observe a larger region of the sky. For the sake of simplicity, the anti-Sun direction is chosen here. Our simulations show that going from 45 deg from the antiSun direction to the anti-Sun direction leads to a decrease in the zodiacal emission of 15\%. For the Darwin/TPF mission, with the Emma X-array configuration, the satellite was limited to an annular region on the sky between $46 \mathrm{deg}$ and $83 \mathrm{deg}$ from the antiSun direction. ${ }^{27}$

- Exozodiacal Emission. An exozodiacal disk is the analog to the local zodiacal disk but associated with the exoplanetary system. The presence of an exozodiacal disk, depending on its inclination and emission, creates a flux that could be higher than the planetary signal. ${ }^{17,32}$ It means that an exoplanet would be harder to detect in this situation. However, recent results from the Large Binocular Telescope Interferometer (LBTI) suggest that the median level of the exozodiacal emission around nearby stars is below 27 zodis (95\% confidence; 1 zodi corresponds to the level of the zodiacal emission in the solar system) for a sample of stars without cold dust disk (i.e., Kuiper belt analog). ${ }^{33}$ In our study, the planet population synthesis tool generates an exozodical level based on the measured LBTI statistics. In Fig 3, the exozodiacal disk emission for the putative exoplanet system is represented. The corresponding level is 3.7 zodis (Table 2). Our exozodiacal model is based on the DIRBE interplanetary dust model. ${ }^{31}$ The bulge around $2 \mu \mathrm{m}$ is due to the scattering of the dust. Nevertheless, the emission of this dust is negligible compared to stellar leakage or the local zodiacal dust emission at short wavelengths $(<5 \mu \mathrm{m})$ and instrumental background at long wavelength $(>5 \mu \mathrm{m})$. In this example, the dust temperature is only $62 \mathrm{~K}$ since it is based on the luminosity of the star (Proxima Centauri has a bolometric luminosity of $0.0017 \mathrm{~L}_{\odot}$ ).

- Instrumental Background Flux. The Sun and the Earth (in low Earth orbit) radiate energy toward the satellite. The baffle of each aperture, as well as the attitude of the satellite, will prevent any direct solar light entering the interferometer. We assume that the telescopes and optical train radiate as a grey body with an emissivity of 0.25 .

- Instrumental Throughput. The instrumental throughput is an important figure of merit since only a few photons are received from the exoplanet at the telescope aperture (i.e., $\approx 2.8 \mathrm{ph} / \mathrm{s}$ for the selected putative planet, with $D=0.25 \mathrm{~m}$, at $2.5 \mu \mathrm{m}$ and 
$R=1.2$, see Sec. 3.2). This parameter is the multiplication of the reflectance of the mirrors of each aperture, the fiber coupling efficiency, and the quantum efficiency $(\mathrm{QE})$ of the detector. The assumption is that each aperture is a classical reflective telescope with three mirrors, without central obscuration. Both apertures of the interferometer focus the light into single-mode optical fibers of same length to avoid any chromatic dispersion. ${ }^{34}$ They were not present in the Bracewell proposal, but their addition leads the correction of phase defects of the incoming wavefront, except a relative piston. ${ }^{6,35}$ Each single-mode waveguide has a coupling efficiency, $\rho_{i}$, and only the fundamental mode is propagated if we work close to or below the cutoff frequency. ${ }^{36}$ This fundamental mode can be approximated by a Gaussian pattern and the circular pupil creates an Airy pattern on the focal plane. Due to the mismatch between both profiles, the coupling efficiency reaches a maximum value of $\approx 81 \%$ and not $100 \%$. A real fiber tends to achieve this value, but due to approximations (not a pure Gaussian pattern) and due to Fresnel reflections on the fiber head, it is reduced to a maximum of $78 \% .{ }^{37}$ Any aberrations, geometrical or physical, like a tip/tilt or a central obscuration, decrease the coupling efficiency and can lead to very low transmission. ${ }^{35}$ The detectors used for this application are two photodiodes since only two pixels are needed. Unfortunately, the detector QE is not constant over a large bandwidth since it depends on the detector technology. To keep a pure photonic case, specific detector properties are not considered. Although still noisy, compared to optical detectors, infrared technology evolves quite rapidly and due to the timing to build such a mission, making a detector choice at this stage seems irrelevant. However, we assume a generic value of the QE of $80 \%$ not to overestimate our results. ${ }^{38}$ It is a typical value for infrared detector arrays used in astronomy. The instrumental throughput is estimated to be around 50\% at the shortest wavelength and $30 \%$ at the longest wavelength of the bandwidth. This bandwidth is introduced in the next section. This variation of instrumental throughput is due to the intrinsic drop of coupling efficiency over a large bandwidth. No instrumental degradation, as a tip/tilt angle, is considered here. Once taken into account, it will reduce the instrumental throughput.

\subsection{Noise Determination}

Figure 4 represents the planet signal (ph/s) and two noise contributors, the shot noise and the instrumental noise, in the detector plane, from 0.5 to $30 \mu \mathrm{m}$ (PROBA-size configuration, cf., Table 1) for the synthetic planet. Based on the following discussion, the tip/tilt angle and the root-mean-square (RMS) OPD are, respectively, set to 300 mas and $10 \mathrm{~nm}$. To obtain these curves, the null depth is determined as well as all noise contributors that play a role in the SNR (cf., Sec. 4).

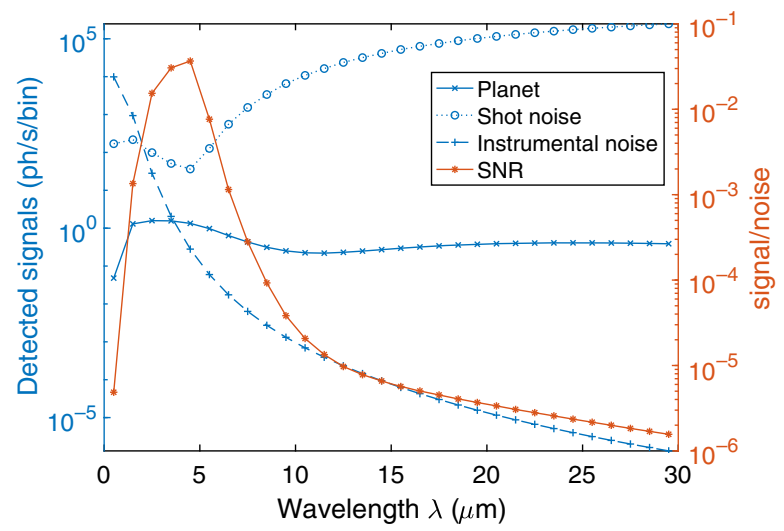

Fig. 4 Planet signal (ph/s) and two noise contributors (left axis) and SNR (right axis) of a synthetic planet (parameters from Table 2) around Proxima Centauri. The resolution parameter is set to $R=1.2$. It corresponds to a $50 \%$ loss of coupling efficiency at the longest wavelength. PROBAsize configuration: $B=5 \mathrm{~m}, D=0.25 \mathrm{~m}$, and $T=100 \mathrm{~K}$. The RMS differential tip/tilt is set to 300 mas and the RMS OPD to $10 \mathrm{~nm}$. The baseline length is not optimized for this specific planet. 
A spectral bandwidth integrates the incoming signals over multiple wavelengths. Over a bandwidth of $\left[\lambda_{c}, 2.4 \lambda_{c}\right]$, with $\lambda_{c}$ the cutoff frequency of the fiber, we derived that the coupling efficiency is decreased by $\approx 50 \%$ due to chromatic coupling effects at the longest wavelength. This bandwidth corresponds to a resolution parameter $R$ of $1.2(=\lambda / \Delta \lambda)$. A larger $R$ in the infrared domain would lead to low-resolution spectrometry and, of course, less flux per spectral channel. We are interested here in the planet detection and not their full characterization.

\subsubsection{Null depth}

The null depth is defined as

$$
N=\frac{I_{-}}{I_{+}}
$$

where $I_{+}$is the intensity at the constructive output and $I_{-}$at the destructive one. ${ }^{39} \mathrm{It}$ is one of the main figures of merit of nulling interferometry. By operating at the null phase, the noise is minimal in comparison to standard interferometry. ${ }^{40}$ This null depth can be decomposed as a timeaveraged null depth $\bar{N}$ in the presence of active OPD matching and intensity balancing, and an RMS fluctuation of the null level $\sigma_{N}{ }^{39}$

The time-averaged null depth of a Bracewell interferometer observing a single star is given by

$$
\bar{N}=\frac{1}{4}\left[\sigma_{\phi}^{2}+\frac{\pi^{2}}{4}\left(\frac{\theta_{\mathrm{dia}}}{\lambda_{\mathrm{sh}} / b}\right)^{2}+\sigma_{I}^{2}\right]
$$

if the contribution from polarization is neglected and the phase dispersion across the passband is considered constant in vacuum. ${ }^{39} \sigma_{\phi}^{2}$ is the variance of the phase error. The second term $\frac{\pi^{2}}{4}\left(\frac{\theta_{\mathrm{dia}}}{\lambda_{\mathrm{sh}} / b}\right)^{2}$ is related to the finite extent of the star and leads to a "stellar leakage" in the destructive output with $\theta_{\mathrm{dia}}$ is the star angular diameter, $b$ is the baseline length, and $\lambda_{\mathrm{sh}}$ is the shortest wavelength in the bandwidth. $\sigma_{I}^{2}$ is the variance of the fractional intensity deviations. ${ }^{39}$ This equation contributes to the mean residual stellar flux. The RMS fluctuation of the null level is given by ${ }^{39}$

$$
\sigma_{N}=\sqrt{\frac{\sigma_{\phi}^{4}+\sigma_{I}^{4}}{8}} .
$$

Impact of $\sigma_{\phi}^{2}$ and $\sigma_{I}^{2}$ parameters are discussed in Sec. 4. Thanks to the single-mode optical fiber, any wavefront errors are converted into intensity errors $\sigma_{I}$ at the output, which are less severe than a pure phase effect on the nulling. Photometric imbalance from the stellar leaks (residual stellar flux) is of the second order as compared with phase aberrations. ${ }^{35}$

\subsubsection{Shot noise}

The shot noise is directly related to the incoming signals and associated fluxes. It contributes as

$$
N_{s}=\sqrt{\mathrm{OPF}+\mathrm{OBF}+\mathrm{OSF}}
$$

in $\mathrm{ph} / \sqrt{\mathrm{s}}$ with $\mathrm{OPF}$ is the output planet flux $(\mathrm{ph} / \mathrm{s}), \mathrm{OBF}$ is the output background flux $(\mathrm{ph} / \mathrm{s})$, and OSF is the Stellar output flux (ph/s).

$\mathrm{OPF}$ is the planet flux multiplied by the instrumental throughput and the transmission map. If the planet is unfortunately located on a dark fringe, the flux is not transmitted, whereas it is fully transmitted if the planet is on a bright fringe. If the goal is to observe only one specific known exoplanet, its position in time and space can be computed. The baseline length is optimized as well as the orientation to get a maximum of transmission. For a more general case, when planet's 

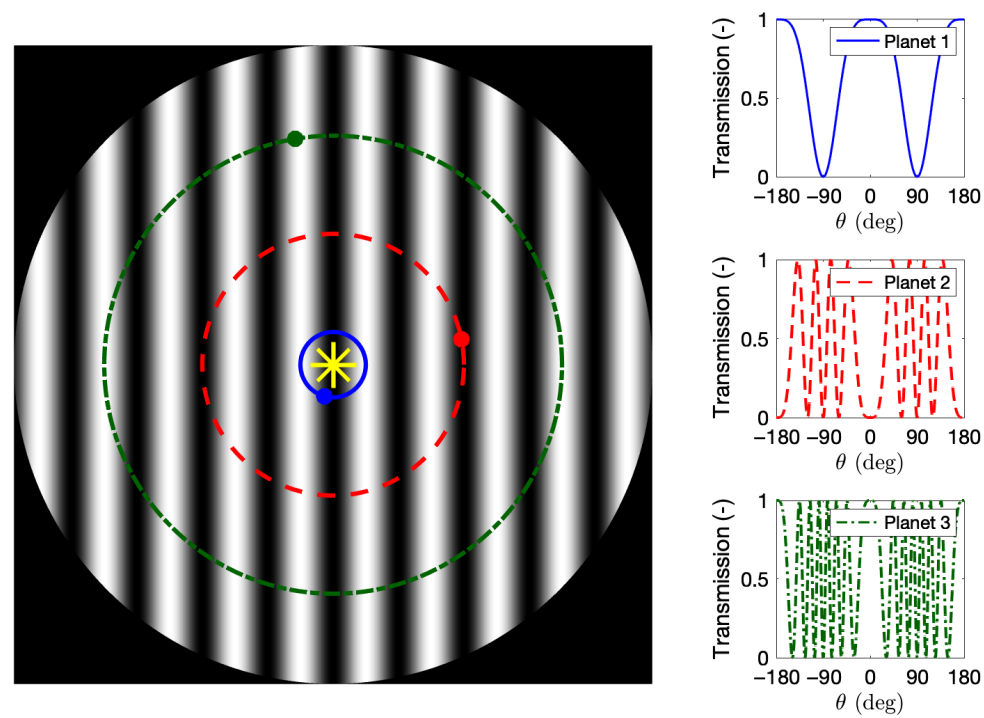

Fig. 5 Transmission map of a typical nulling Bracewell interferometer. The interferometer rotates and hypothetical planets $(1,2$, and 3$)$ cross bright and dark fringes. The signal (small three figures) is modulated. Reference axis for $\theta$ is the horizontal one, across the fringes.

position is unknown, on average, only $50 \%$ of the flux is transmitted and contributes to the shot noise.

To retrieve the planet from all signals and distinguish it from the background, a solution is to rotate the interferometer around its line-of-sight as proposed by Bracewell. ${ }^{6}$ The planet crosses bright and dark fringes and its flux is modulated. The background, considered as uniform, is not modulated. Figure 5 shows the monochromatic transmission map of a typical nulling Bracewell interferometer. The star is located on the central dark fringe, whereas putative planets can be located anywhere in the sky. With a rotation of the interferometer around the line-of-sight, they cross different fringes, and the transmission is modulated as represented. To retrieve the planet modulated signal, a cross-correlation transform is computed between expected signals and the real modulation of the planet. ${ }^{41}$ This modulation comes nevertheless with a drawback. The measured planet is, on average, reduced to $50 \%$ in the case of a Bracewell interferometer.

OBF represents the contribution of all possible thermal background emissions that can limit the detection of an exoplanet. Here are considered the local zodiacal dust emissions, the exozodiacal dust emissions and, the instrumental thermal emission.

OSF is the residual part of the stellar flux, which is not rejected. Therefore, it is simply equal to the stellar flux multiplied by $\bar{N}$ from Eq. (2).

\subsubsection{Instrumental nulling noise}

The instrumental stellar leakage $N_{\text {inst }}$ is the multiplication of the $\sigma_{N}$ term [Eq. (3)] by the OSF. It is a noise directly linked to the stability of the null and how it can be controlled. It depends on the co-phasing error (OPD) and the mismatches of intensities (tip/tilt) at the beam recombination.

\section{Signal-to-Noise Ratio}

To retrieve a modulated planet signal, the minimal needed SNR is fixed to 5. During the acquisition, the spacecraft performs a whole number of rotations. This SNR is expressed per second and leads to the computation of the integration time. If the orbital parameters of the planet are known a priori (from indirect methods), one can compute the total integration time to get a requested $\mathrm{SNR}_{\text {req }}$ of 5 . In the case of an unknown planet, one needs to fix a maximum allowed integration time. It is fixed by the architecture constraints as well as the platform stability in time. The SNR generated during $1 \mathrm{~s}\left(\mathrm{SNR}_{1 \mathrm{~s}}\right)$ is given by 


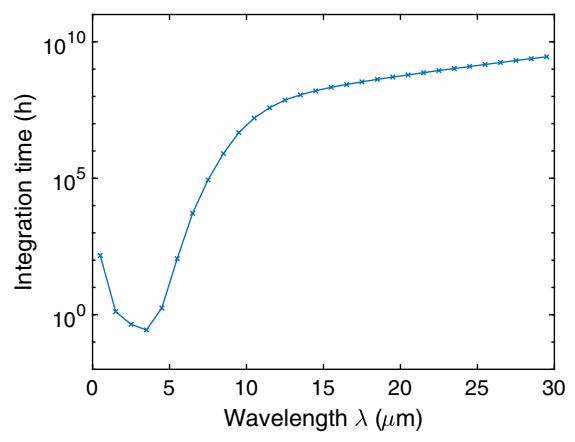

Fig. 6 Integration time of a synthetic planet (parameters from Table 2) around Proxima Centauri. The resolution parameter is set to $R=1.2$. It corresponds to a $50 \%$ loss of coupling efficiency at the longest wavelength. PROBA-size configuration: $B=5 \mathrm{~m}, D=0.25 \mathrm{~m}$, and $T=100 \mathrm{~K}$. Platform stability constraints (tip/tilt and OPD) are not considered.

$$
\mathrm{SNR}_{1 \mathrm{~s}}=\frac{\mathrm{OPF}}{\sqrt{\left(N_{s}^{2}+N_{\text {inst }}^{2}\right)}}
$$

The detector noise is not considered. The integration time $t_{i}$ for a minimal required SNR $\left(\mathrm{SNR}_{\text {req }}\right)$ is simply equal to

$$
t_{i}=\left(\frac{\mathrm{SNR}_{\mathrm{req}}}{\mathrm{SNR}_{1 \mathrm{~s}}}\right)^{2}=\left(\frac{5}{\mathrm{SNR}_{1 \mathrm{~s}}}\right)^{2}
$$

Figure 6 shows the integration time as a function of wavelength (in the case of the PROBAsize configuration) without considering the instrumental nulling noise. The minimum corresponds to $0.3 \mathrm{~h}$ at $3.5 \mu \mathrm{m}$. It rises rapidly at long wavelengths due to the thermal background. As shown in Eq. (6), the integration time is the inverse of the SNR. Therefore, the integration time curve shape (Fig. 6) is the inverse SNR curve shape (Fig. 4).

\subsection{Impact of Tip/Tilt}

In this section, the effect of tip/tilt is investigated. Two cases are possible, a global and differential one. The global tip/tilt, so when the spacecraft does not point perfectly at the star and wobbles around it, is not treated here. In addition to a fine attitude controller on the platform, tip/tilt mirrors will be used to correct this mode. They are anyway needed to compensate for the differential tip/tilt angles as explained below.

In the case of a differential tip/tilt, the satellite has a zero-mean tip/tilt angle, and we introduce an RMS differential tip/tilt angle between both pupils. During the integration time, the two pupils are, therefore, misaligned by this angle, which is converted to a translation of the PSF in the image plane, i.e., at the tip of the fiber. ${ }^{37}$ We compute the coupling efficiency loss numerically by the overlap integral of the fundamental mode of the fiber and the tilted PSF. The instrumental throughput is, therefore, different in both apertures. It creates an intensity imbalance at the output. This value $\sigma_{I}$ affects both the time-average null depth [Eq. (2)] and the RMS fluctuations of the null level [Eq. (3)]. Figure 7 represents the effect of relative tip/tilt between the two pupils up to 0.5 arc sec for the CubeSat, the PROBA-size, and the FKSI-concept configurations. The optimum wavelength, so without any instrumental nulling noise, is selected for each platform. It means that the dependence on wavelength is not shown. As expected, the integration time is severely affected by an increase of the relative tip/tilt angle. One can see that the diameter size has a non-negligible impact. When the diameter is increased, the Airy disk size is reduced, and the coupling efficiency with the fiber is than severely affected by a small RMS differential tip/tilt angle.

From this figure, one can derive the value used for the PROBA case to stay below an integration time of $24 \mathrm{~h}$ (dotted black line). 300 mas is the upper limit. This value was the one selected in Fig. 4. 




(a)

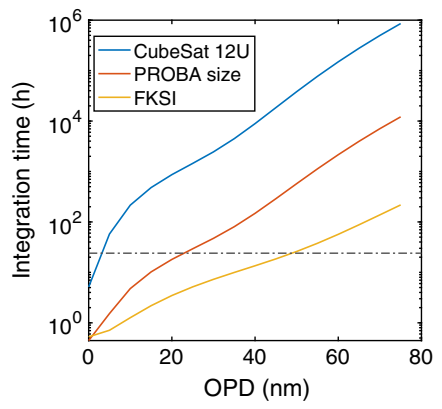

(b)

Fig. 7 Integration time of a synthetic planet (parameters from Table 2) around Proxima Centauri as a function of (a) the RMS differential tip/tilt angle and (b) OPD. The dotted black line represents an arbitrary integration time of $24 \mathrm{~h}$. The resolution parameter $R=1.2$. The selected wavelength is the optimum wavelength for each configuration (Table 4). CubeSat 12U, PROBA-size, and FKSIconcept configurations (parameters from Table 1).

\subsection{Impact of the OPD}

Another instrumental parameter to take into account is the OPD. Only the first-order OPD, so a pure piston, is considered in this study. Higher-order OPD changes will be corrected by the single-mode waveguide and converted in an intensity imbalance at the output of the fibers. Figure 7 represents the variation of the integration time for the synthetic planet as a function of RMS OPD. Requirements are very stringent and only a few $\mathrm{nm}$ of OPD $(<35 \mathrm{~nm})$ is allowed to keep reasonable integration time $(\approx 24 \mathrm{~h})$ for the FKSI-concept or PROBA-size satellites. It proves that good OPD sensors and very fine OPD corrections are necessary. Note that the OPD impact is wavelength-dependent since $\sigma_{\phi}^{2}$ from Eq. (2) equals $\left(2 \pi \sigma_{\mathrm{OPD}} / \lambda\right)^{2} . \sigma_{\mathrm{OPD}}$ is the value represented in Fig. 7. As the optimum wavelength for each configuration is considered here, this dependence is not shown.

\section{Exoplanet Missions Yield}

In this section, four types of missions are considered (Table 1). The P-POP algorithm, described below, is used to generate a set of synthetic planets around 326 real nearby main-sequence stars (distance $<20 \mathrm{pc}$ ). Depending on their characteristics (size, orbit, and star), our algorithm computes the integration time needed to detect them for each satellite configuration. Some intrinsic characteristics of these configurations are analyzed, as the optical chain temperature, the OPD, or the tip/tilt accuracy.

\subsection{P-POP Algorithm}

The Kepler satellite, from NASA, discovered more than half of the current exoplanet population thanks to the transit method. ${ }^{42}$ Occurrence rates of exoplanets were derived, based on their size, orbital period, and stellar host type. These occurrence rates are aggregated in the SAG13 model from NASA ${ }^{43}$ From these, we generate synthetic planet populations using the Monte-Carlo tool (P-POP) described in Kammerer and Quanz ${ }^{16}$ around 326 real main-sequence stars located within a radius of $20 \mathrm{pc}$ around the Earth. ${ }^{44}$ Around each star, we draw 100 planetary populations (or universe) from our occurrence rate distribution, yielding a statistically robust sample of $\approx 86,000$ planets in total. Some stars can have multiple planets, whereas some have no planet. The total number of synthetic planets in a planet radius-stellar insolation plot is shown in Fig. 8. The mean value is shown as well as its standard deviation. This standard deviation is around $10 \%$. It is related to error of a Poisson-like distribution and is coherent with 100 trials. P-POP generates mainly terrestrial planets (below $4 R_{\oplus}$ ) with stellar insolation (normalized) close to that of the Earth. This kind of planet is expected to be the most common. ${ }^{3}$ 
Dandumont et al.: Exoplanet detection yield of a space-based Bracewell interferometer...
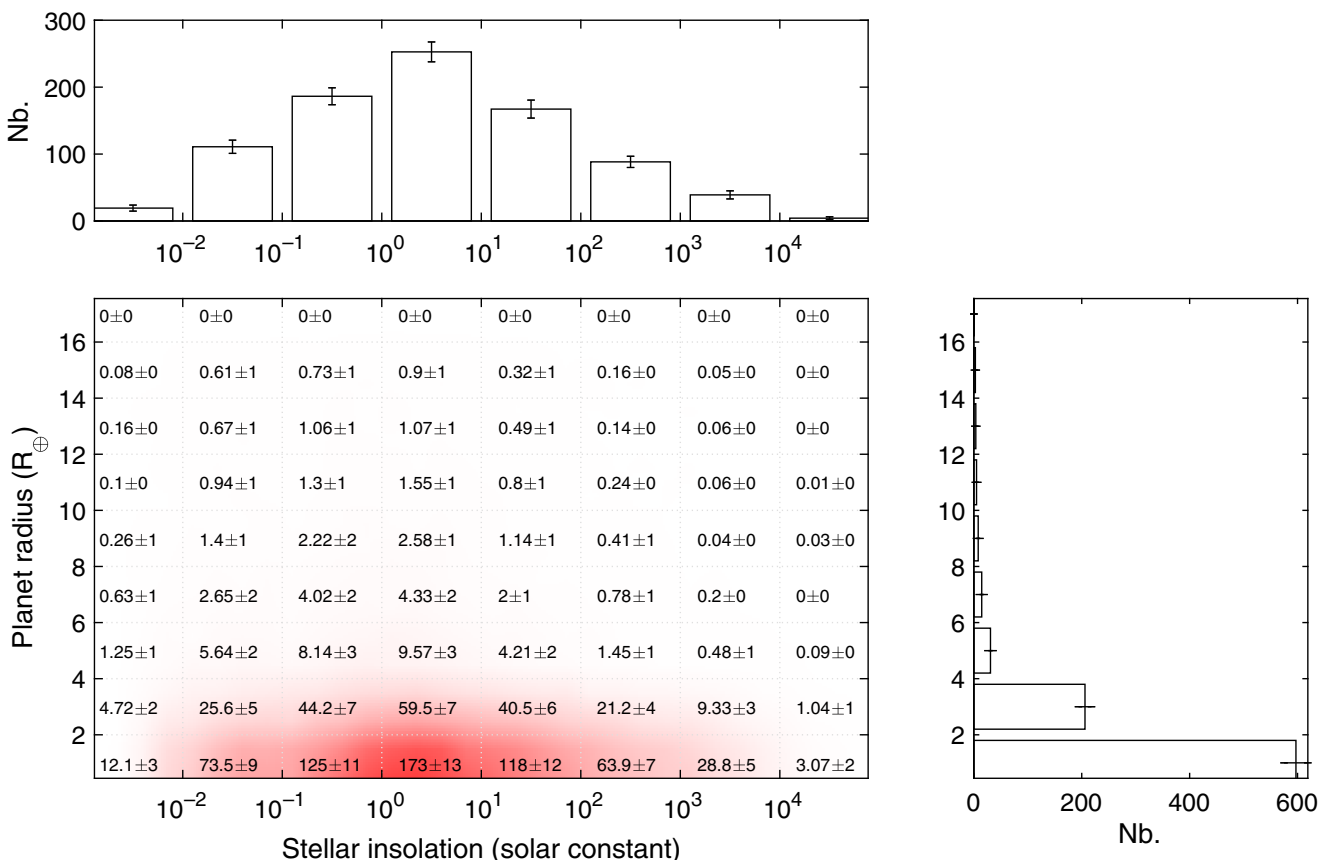

Fig. 8 Mean generated P-POP population (100 trials around 326 real stars) and standard deviation. Redder (darker) region corresponds to more occurrences. Each histogram is the sum of the synthetic planets in the columns or lines of the central figure.

\subsection{Operating Wavelength}

One of the main instrumental parameters of an interferometer is the operating wavelength. Even if the mid-IR ( 3 to $20 \mu \mathrm{m}$ ) is the one that it is envisaged for a large interferometric mission, ${ }^{10}$ it is not the optimal one for a smaller mission. The central wavelength needs to be adapted and, there is a trade-off to make between scientific interests and the operating wavelength. Figure 9 represents the mean total number of detections per P-POP sample from 0.5 to $20 \mu \mathrm{m}$ for each configuration (Table 1). It covers the visible and infrared domains. The integration time spent on each star is set to a maximum of $24 \mathrm{~h}$, and we count each planet that reaches an SNR of 5 during that time. This maximum integration time value, and how it affects the results, is discussed below (Sec. 5.4). As expected, CubeSats (6U to 12U) could detect only a few planets (around 5 to 15 planets) while the PROBA-size architecture, even being a small satellite, could detect 120 planets

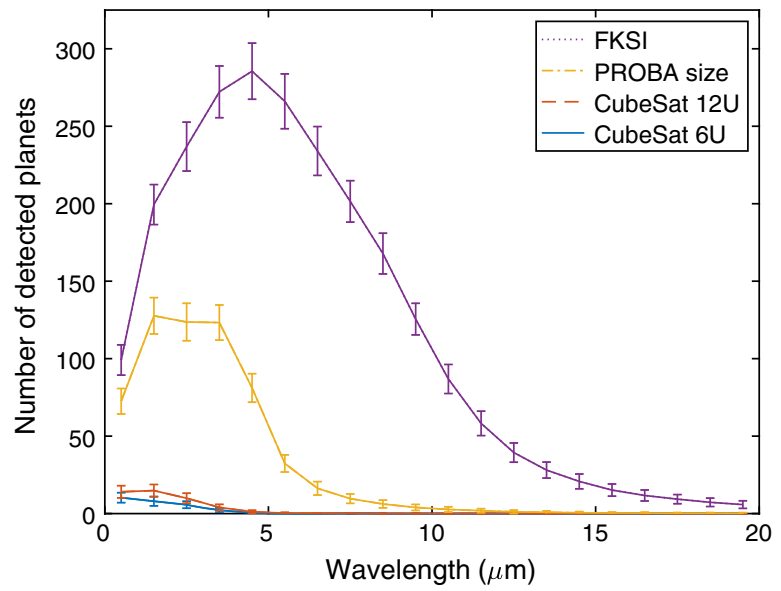

Fig. 9 Number of detected planets with the four configurations defined in Table 1 with at least a SNR of 5 and a integration time of $24 \mathrm{~h}$. Platform stability constraints (tip/tilt and OPD) are not considered. The resolution parameter $R=1.2$. The error bars represent the standard deviation. 
around $2.5 \mu \mathrm{m}$. FKSI, as a NASA Discovery proposal mission, could detect around 285 planets at $4.5 \mu \mathrm{m}$. One has to keep in mind that no platform stability constraints (tip/tilt and OPD) were considered in these simulations. Their effects are discussed in Secs. 5.5 and 5.6. A table with all statistical relevant information (detections per star type, spectral type, radius, distance, etc.) is available in Table 3. From Fig. 9, the optimal central wavelength can be computed (available in Table 3). Let us recall that from each value represented, the bandwidth is already considered as the $R$ parameter is set to 1.2 (50\% loss of coupling efficiency) as discussed in Sec. 3.2. The peak for each configuration is directly related to the peak of the SNR for one planet (Fig. 4). Since every planet is different, the peak is wider, especially in the PROBA size case.

\subsection{Instrumental Temperature Effect}

The instrumental temperature is the main noise contributor at long wavelength, as already shown in Fig. 3. It drastically limits the number of detected planets. In Fig. 9, the FKSI-concept curve is shifted toward longer wavelengths because of the lower instrument temperature and the resultant shift of the instrumental blackbody emission to longer wavelengths. Figure 10 represents the number of detected planets from PROBA-size case with different instrumental background temperatures. The wavelength domain is shortened to 0.5 to $15.0 \mu \mathrm{m}$ to better see the effect. The maximum number of detections decreases when the temperature increases, even at constant wavelengths.

\subsection{Integration Time Effect}

The integration time is an important parameter for this mission. Figure 10 represents the number of detected planets for each configuration at their optimum wavelength as a function of the maximum integration time per target. The satellite points to the star during that time and collects photons, expecting to find a planet. The $6 \mathrm{U}$ CubeSat was omitted for better clarity. For the $12 \mathrm{U}$ CubeSat, by doubling the time spent on each star, from 12 to $24 \mathrm{~h}$, the number of detections goes from 5 to 7.5. The curve quickly reaches a plateau. The integration time is not a limiting factor as the SNR will not increase sufficiently quickly with time to detect more exoplanets. For PROBA or FKSI, increasing the integration time leads to a net increase of the detection yield. It clearly shows that these missions need to stay few hours, typically $24 \mathrm{~h}$, on each star to not "miss" many exoplanets. The optimum integration time needs to be based on a full study of the spacecraft stability during a long period as well as the expected lifetime of the satellite. It will give us confidence in the maximum allowed integration time.

\subsection{Tip/Tilt Effect}

As shown in Sec. 4.1, the differential tip/tilt has a huge effect on the integration time. Figure 11 represents the number of detected planets as function of the RMS differential tip/tilt angle (pupils misaligned-zero-mean error). The optimum wavelength for each configuration is selected, and $24 \mathrm{~h}$ is used as integration time. The relative tip/tilt is a big issue since with an angle of only 0.7 arc sec no detection is possible with any configuration. However, in past studies, it was estimated that the tip/tilt could be corrected up to 20 mas for FKSI. ${ }^{27}$ In that case, it leads to a drop of $0.3 \%$ exoplanets, which is negligible.

\subsection{OPD Effect}

As presented for the synthetic planet, the OPD parameter needs to be controlled very accurately. Figure 11 represents the number of detections for each configuration with a varying OPD. As expected, it drops drastically after a few $\mathrm{nm}$. In the case of FKSI, a value of $2 \mathrm{~nm}$ was estimated in past studies ${ }^{27}$ and it gives a drop of $\approx 10$ detections $(\approx 5 \%)$. In fact, $\sigma_{\phi}^{2}$ acts on the time-average null depth and the RMS fluctuations of the null level [Eqs. (2) and (3)]. The instrumental nulling noise is increased, leading to a drop of SNR and so a drop of detections at constant integration time. 


\subsection{Summary of Results}

Table 3 summarizes all the important statistical information about the detected exoplanet population. For these values, 100 trials were used to increase the confidence in the model. 86,000 planets were generated and analyzed.

Each mission detects different planet types based on their physical parameters. Figure 12 shows the radius/stellar insolation variations for each configuration. The mean detection value of each bin is shown. The radius is not the limiting factor, but the stellar insolation is. A CubeSat, as expected, could barely detect exoplanets (Neptunian ones) while FKSI is well suited to detect warm planets (between $10^{1}$ to $10^{3}$ stellar insolation-typical equilibrium temperature of $760 \mathrm{~K}$ ). It is consistent with the results of $2010 .{ }^{46} \mathrm{~A}$ sample of our results is available in Appendix $\mathrm{C}$.

Our exoplanet yield predictions can be compared to other space-based missions using direct imaging methods. However, it is always a difficult task to make a fair comparison between missions since hypotheses are not the same (no platform stability constraints here). Moreover, different technologies (coronagraphy versus interferometry) lead to different working wavelengths (optical/NIR to MIR).

For a large mid-infrared interferometer, Kammerer and Quanz (2018) have estimated the yield to 315 exoplanets using the same exoplanet population synthesis tool as in this paper, and an

Table 3 Statistical information (100 synthetic planet populations) about the four configurations from Table 1.

\begin{tabular}{|c|c|c|c|c|}
\hline & CubeSat $6 U$ & CubeSat $12 U$ & PROBA-size & $\begin{array}{c}\text { FKSI } \\
\text { concept }\end{array}$ \\
\hline Baseline length $(\mathrm{m})$ & 0.5 & 1 & 5 & 12.5 \\
\hline Pupil diameter (m) & 0.08 & 0.08 & 0.25 & 0.5 \\
\hline Optical train temperature $(\mathrm{K})$ & 150 & 150 & 100 & 60 \\
\hline Optimal wavelength $(\mu \mathrm{m})$ & 0.5 & 1.5 & 2.5 & 4.5 \\
\hline Waveband $(\mu \mathrm{m} ; R=1.2)$ & 0.3 to 0.7 & 0.9 to 2.1 & 1.5 to 3.5 & 2.6 to 6.4 \\
\hline Mean number of detections & $10 \pm 3$ & $15 \pm 4$ & $120 \pm 12$ & $280 \pm 18$ \\
\hline Median number of detections & 10 & 15 & 123 & 286 \\
\hline Mean integration time $(<24 \mathrm{~h})$ and $\mathrm{SNR}=5$ & $9.0 \pm 2.3$ & $8.3 \pm 2.0$ & $6.7 \pm 0.6$ & $4.4 \pm 0.3$ \\
\hline Median radius $\left(R_{\oplus}\right)$ & 8.1 & 6.7 & 3.7 & 2.6 \\
\hline Mean number of "rocky" $R<1 R_{\oplus}$ & 0.12 & 0.48 & 6.0 & 51 \\
\hline Mean number of "super-Earths" $1.0 R_{\oplus}<R<1.75 R_{\oplus}$ & 0.56 & 0.98 & 20 & 76 \\
\hline Mean number of "sub-Neptunes" $1.75 R_{\oplus}<R<3.5 R_{\oplus}$ & 2.0 & 4.5 & 64 & 120 \\
\hline Mean number of "Neptunes" $M>130 M_{\oplus}$ & 1.1 & 1.9 & 14 & 19 \\
\hline Mean number of "Jovians" $M>130 M_{\oplus}$ & 6.5 & 7.0 & 20 & 22 \\
\hline Mean number of planets around AFGK stars & 9.7 & 12 & 90 & 230 \\
\hline Mean number of planets around $\mathrm{M}$ stars & 0.6 & 2.7 & 34 & 56 \\
\hline Mean temperature $(\mathrm{K})$ & $770 \pm 260$ & $820 \pm 230$ & $850 \pm 60$ & $780 \pm 30$ \\
\hline Min distance $(p c)$ & 3.9 & 3.8 & 9.0 & 11 \\
\hline Mean distance $(p c)$ & 8.2 & 6.1 & 10.0 & 12 \\
\hline
\end{tabular}

A detailed figure about the distribution over universes is available in Appendix $A$. The planet classification is based on Ref. 45. A detailed figure about this classification is available in Appendix $\mathrm{B}$. The $R$ parameter is selected to have a $50 \%$ coupling efficiency drop over the bandwidth. No platform stability constraints (tip/tilt and OPD) are considered at this stage for both figures. Their effects are discussed in Secs. 5.5 and 5.6. 


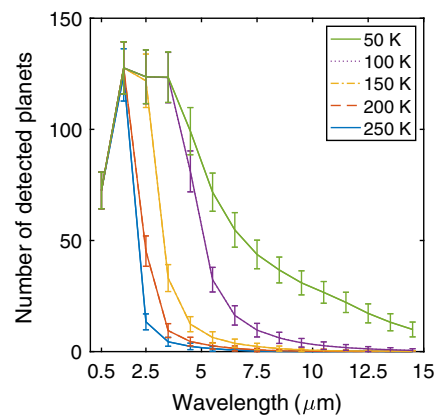

(a)

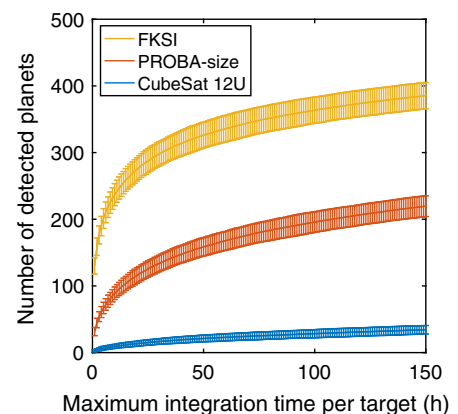

(b)

Fig. 10 (a) Number of detected planets with the PROBA-size configuration (Table 1) with an instrumental temperature variation. (b) Number of detected planets with three configurations (Table 1) as a function of maximum integration time per target. The selected wavelength is the optimum wavelength for each configuration (Table 3). The CubeSat $6 \mathrm{U}$ was omitted for clarity. No platform stability constraints (tip/tilt and OPD) are considered at this stage for both figures. Their effects are discussed in Secs. 5.5 and 5.6. The error bars represent the standard deviation.

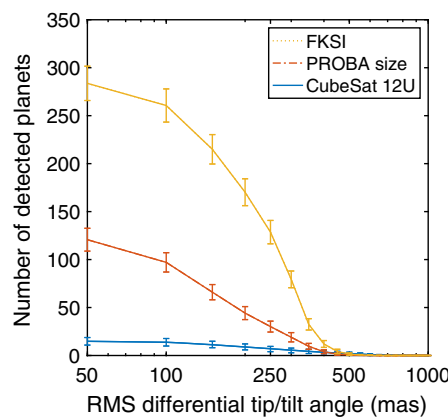

(a)



(b)

Fig. 11 Number of detected planets with three configurations (Table 1) as a function of (a) the RMS differential tip/tilt angle (pupils misaligned-zero-mean error) and (b) of the OPD. The selected wavelength is the optimum one for each configuration (Table 3). The integration time is set to $24 \mathrm{~h}$. The CubeSat $6 \mathrm{U}$ was omitted for clarity. The error bars represent the standard deviation.

integration time of 35,000 s (compared to 86,400 s in Table 3). Figure 10 shows that the yield for the FKSI-concept is about 236 exoplanets with the same integration time. The collecting area is, of course, different. Our FKSI-concept is composed of two principal mirrors of $0.5 \mathrm{~m}$, whereas the mid-infrared interferometer is composed of four mirrors of $2.8 \mathrm{~m} .{ }^{16}$ With a smaller collecting area and a smaller baseline length ( $12.5 \mathrm{~m}$ compared to $168 \mathrm{~m}$ ), our FKSI-concept is more focused on hotter exoplanets and is not optimized to detect "Terran" worlds. Moreover, no platform stability constraints (tip/tilt and OPD) are considered for our value and, the waveband is different.

They also make comparisons with space-based optical/NIR telescopes, like the NASA HabEx or LUVOIR missions. They estimated the yield to around 207 exoplanets with again an integration time of 35,000 s. ${ }^{16}$ In Ref. 45, it is shown that HabEX or LUVOIR missions have a profile close to the FKSI-concept with a focus on sub-Neptune planets.

\section{Conclusion}

Nulling interferometry is one of the most promising solutions to spectrally characterize exoplanets. In this paper, we investigated four space-based mission architectures using nulling interferometry in a Bracewell configuration, from nano- to medium-satellites. This selection covers a large variety of parameters and leads to the characterization of the potential exoplanet detection yield of these missions. To compute detection yields, we used the P-POP exoplanet synthesis tool combined with an end-to-end instrumental model. To be versatile as possible, the approach is based on a parametric study. Nulling performance is analyzed as a function of the operating 
Dandumont et al.: Exoplanet detection yield of a space-based Bracewell interferometer...

Cubesat 6U

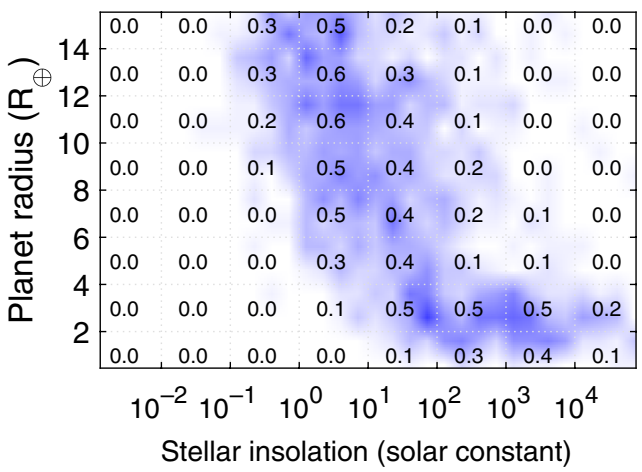

PROBA-size



CubeSat 12U

\begin{tabular}{|c|c|c|c|c|c|c|c|c|}
\hline & 0.0 & 0.1 & 0.3 & 0.5 & 0.2 & 0.1 & 0.1 & 0.0 \\
\hline & 0.0 & 0.1 & 0.5 & 0.4 & 0.2 & 0.1 & 0.0 & 0.0 \\
\hline$=12$ & 0.0 & 0.1 & 0.3 & 0.6 & 0.4 & 0.1 & 0.0 & 0.0 \\
\hline & 0.0 & 0.1 & 0.3 & 0.6 & 0.3 & 0.2 & 0.0 & 0.0 \\
\hline & 0.0 & 0.0 & 0.2 & 0.6 & 0.4 & 0.2 & 0.1 & 0.0 \\
\hline  & 0.0 & 0.0 & 0.2 & 0.5 & 0.5 & 0.2 & 0.2 & 0.0 \\
\hline$\overline{\mathbf{n}}$ & 0.0 & 0.1 & 0.3 & 0.8 & 1.1 & 0.9 & 0.8 & 0.3 \\
\hline & 0.0 & 0.0 & 0.0 & 0.0 & 0.3 & 0.5 & 0.7 & 0.4 \\
\hline & & & & & & & & \\
\hline
\end{tabular}

FKSI concept

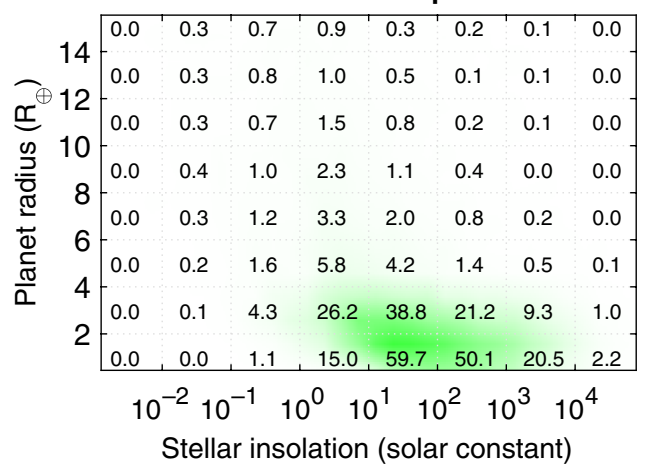

Fig. 12 Mean detection for each of the configuration from Table 1 (diameter, baseline length, and temperature). 100 trials around 326 real stars. The selected wavelength is the optimum one for each configuration (Table 3). No platform stability constraints (tip/tilt and OPD) are considered at this stage for both figures. Their effects are discussed in Secs. 5.5 and 5.6.

wavelength and its spectral bandwidth, the baseline length, the pupil diameter of the two telescopes, the platform stability or the integration time.

Our results show that a PROBA-size mission ( $b \simeq 5 \mathrm{~m} / D \simeq 0.25 \mathrm{~m}$ ) offers the possibility to study about 120 exoplanets, including six warm Terran worlds at $2.5 \mu \mathrm{m}$ without considering any platform stability constraints (tip/tilt and OPD). From this perfect scenario, the RMS differential tip/tilt angle and the RMS OPD have been assessed and studied to derive stability requirements on the platforms. Their impacts on the yield have been investigated, leading to a limit for the RMS differential tip/tilt angle of 200 mas. Therefore, fine compensating mechanisms (e.g., tip/tilt mirrors or ODL) are mandatory. The control of delay in each arm of the interferometer is also the main concern. It leads to stringent requirements in the order of $20 \mathrm{~nm}$.

Positions of these planets were here considered at their maximum elongation (quadrature). However, if the planet orbital position is randomly selected, it leads to a yield drop of $20 \%$ to $50 \%$ depending on the configuration. CubeSats results are really dependent on the planet orbital position (50\% of drop) while FKSI is less impacted (20\%).

Future works will include an instrumental model update considering a full ray-trace design. The objective will be to simulate the optical train, from the apertures to the detector, by considering all individual optical components. With this model, our results will be refined, optical aberrations will be derived, and lead to a more precise mission architecture.

\section{Appendix A: Exoplanet Detection Distributions}

Figure 13 represents the distribution of detections across the universes/trials. It means that, for instance for the PROBA case, there are 30 universes over 100, in which this configuration detects around 120 exoplanets. For each configuration, the mean value and the median value are closed to each other. The number of detections can be seen as a Poisson-like distribution. 

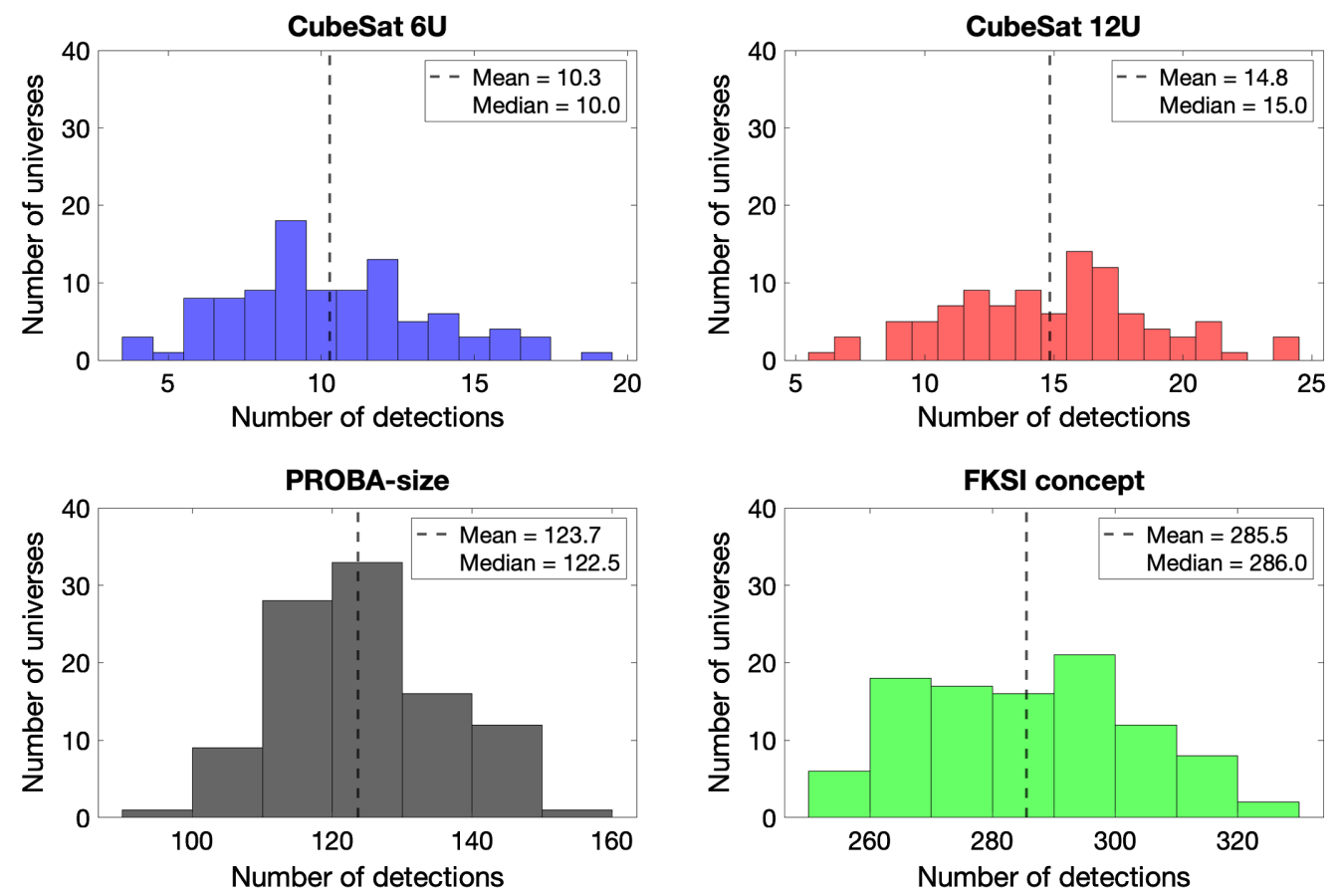

Fig. 13 Distribution of the number of detections across the universes/trials for each configuration (Table 1)

\section{Appendix B: Radius Exoplanet Detection Distributions}

Figure 14 represents the distribution of the mean number of detections per exoplanet type. The exoplanet type is derived from the radius (cf., Table 3) and is based on Ref. 45. As expected, CubeSats detect larger planets (Jovians) and cannot detect rocky planets. A larger mission, as
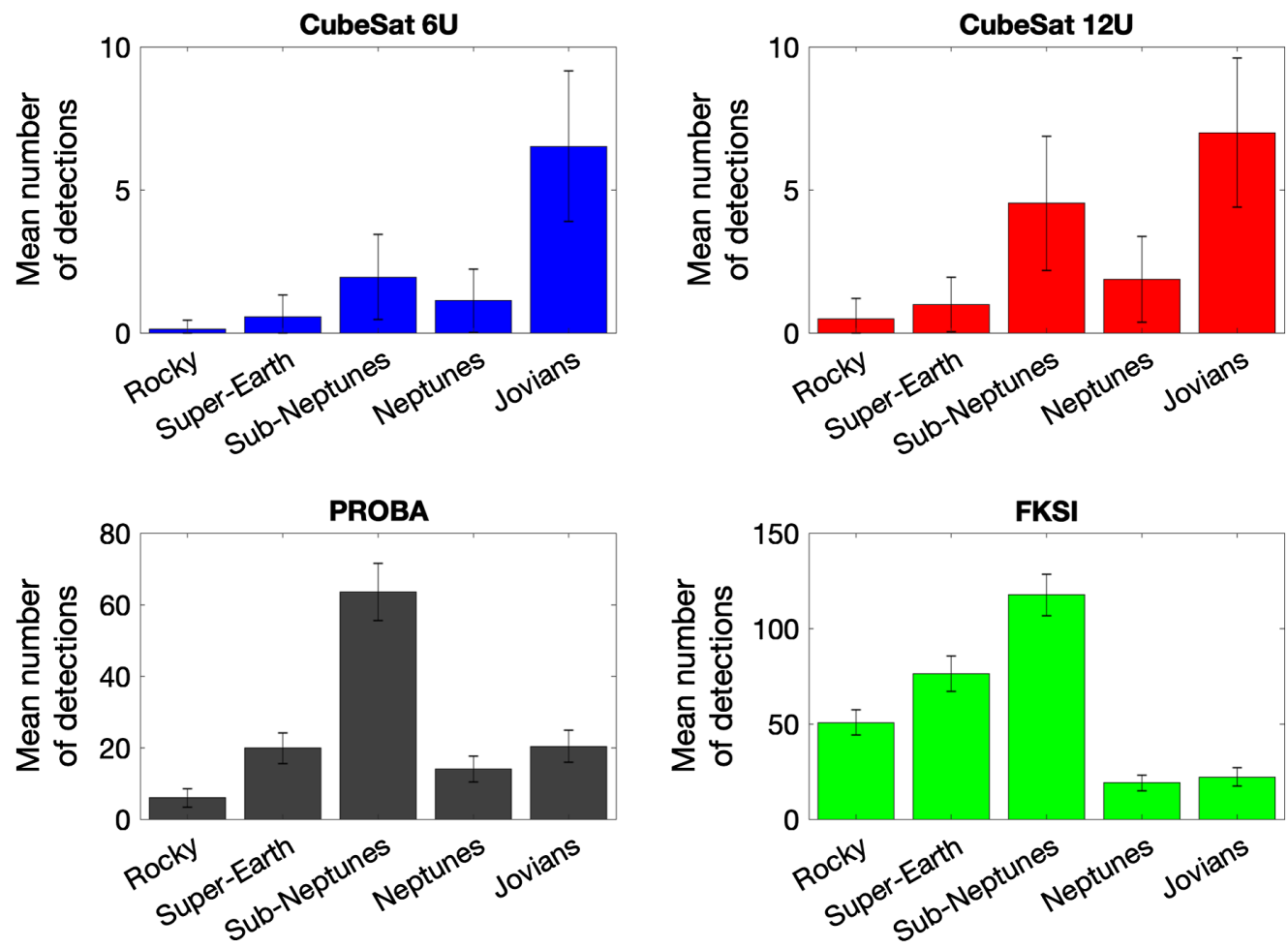

Fig. 14 Mean number of detections for each exoplanet type and for each configuration (Table 1). The exoplanet type is derived from the exoplanet radius. ${ }^{45}$ 
FKSI, is needed for this purpose. The PROBA-like mission is a very versatile configuration, with detection of all types and, a moderate size.

\section{Appendix C: Sample Table}

For each configuration (cf., Table 1), Table 4 shows five randomly selected exoplanets with their related parameters. Full raw data are available online (https://github.com/ColinDandumont/ ExoplanetYield.git).

Table 4 Five randomly selected detected exoplanets for each configuration (Table 1). Only one universe is considered. No platform stability constraints are considered. Full raw data are available online.

\begin{tabular}{|c|c|c|c|c|c|c|c|c|c|c|c|}
\hline & & Star & & & & & & Exoplar & & & \\
\hline $\begin{array}{l}\text { Radius } \\
\left(R_{\text {Sun }}\right)\end{array}$ & $\begin{array}{l}\text { Temper- } \\
\text { ature } \\
(\mathrm{K})\end{array}$ & $\begin{array}{l}\text { Stellar } \\
\text { type }\end{array}$ & $\begin{array}{c}\text { Distance } \\
\text { (pc) }\end{array}$ & $\begin{array}{l}\text { Zodi } \\
\text { level }\end{array}$ & $\begin{array}{l}\text { Radius } \\
\left(R_{\text {Earth }}\right)\end{array}$ & $\begin{array}{l}\text { Temper- } \\
\text { ature } \\
\text { (K) }\end{array}$ & $\begin{array}{l}\text { Semi- } \\
\text { major } \\
\text { axis } \\
(\mathrm{au}) \\
\end{array}$ & $\begin{array}{c}\text { Maximum } \\
\text { angular } \\
\text { separation } \\
\text { (mas) }\end{array}$ & Albedo & $\begin{array}{c}\text { Stellar } \\
\text { insolation } \\
\left(S_{\text {Earth }}\right)\end{array}$ & $\begin{array}{l}\text { Integration } \\
\text { time }(\mathrm{h})\end{array}$ \\
\hline \multicolumn{12}{|c|}{ CubeSat $6 U$} \\
\hline 1.3 & 5900 & $\mathrm{~F}$ & 11 & 130 & 13 & 770 & 0.18 & 17 & 0.20 & 59 & 3.0 \\
\hline 0.72 & 5000 & $\mathrm{~K}$ & 11 & 8.9 & 10 & 170 & 0.77 & 70 & 0.51 & 0.49 & 23 \\
\hline 1.4 & 6200 & $\mathrm{~F}$ & 11 & 62 & 7.2 & 390 & 0.68 & 61 & 0.35 & 6.1 & 21 \\
\hline 1.6 & 5900 & $\mathrm{~F}$ & 11 & 3.5 & 12 & 320 & 0.96 & 88 & 0.49 & 3.2 & 4.4 \\
\hline 1.4 & 6500 & $\mathrm{~F}$ & 15 & 6.1 & 8.4 & 370 & 0.45 & 29 & 0.58 & 15 & 6.7 \\
\hline \multicolumn{12}{|c|}{ CubeSat $12 \mathrm{U}$} \\
\hline 0.14 & 3100 & M & 1.3 & 3.7 & 9.0 & 100 & 0.28 & 210 & 0.39 & 0.020 & 4.1 \\
\hline 0.69 & 4400 & $\mathrm{~K}$ & 5.9 & 56 & 5.8 & 200 & 0.58 & 97 & 0.58 & 0.50 & 9.6 \\
\hline 1.1 & 5700 & $\mathrm{G}$ & 8.4 & 54 & 5.5 & 550 & 0.22 & 27 & 0.29 & 23 & 20 \\
\hline 1.0 & 5400 & $\mathrm{G}$ & 9.1 & 5.6 & 4.8 & 2000 & 0.016 & 1.8 & 0.046 & 3100 & 21 \\
\hline 0.39 & 3500 & M & 2.5 & 23 & 4.9 & 150 & 0.28 & 110 & 0.23 & 0.26 & 13 \\
\hline \multicolumn{12}{|c|}{ PROBA } \\
\hline 1.0 & 5400 & $\mathrm{G}$ & 9.1 & 5.6 & 4.8 & 2000 & 0.016 & 1.8 & 0.046 & 3100 & 0.019 \\
\hline 1.9 & 7500 & A & 5.1 & 15 & 2.2 & 1300 & 0.085 & 17 & 0.16 & 1400 & 0.046 \\
\hline 0.5 & 3700 & M & 3.6 & 9.6 & 5.2 & 180 & 0.43 & 120 & 0.47 & 0.23 & 15 \\
\hline 1.9 & 7500 & $A$ & 5.1 & 15 & 2.0 & 1100 & 0.16 & 31 & 0.25 & 400 & 0.061 \\
\hline 0.4 & 3500 & M & 4.8 & 150 & 2.7 & 180 & 0.19 & 39 & 0.26 & 0.60 & 24 \\
\hline \multicolumn{12}{|c|}{ FKSI } \\
\hline 0.81 & 5100 & $\mathrm{~K}$ & 14 & 65 & 1.3 & 550 & 0.15 & 11 & 0.20 & 17 & 6.0 \\
\hline 0.60 & 4000 & $\mathrm{~K}$ & 3.5 & 13 & 1.1 & 450 & 0.085 & 25 & 0.54 & 11 & 0.46 \\
\hline 1.4 & 6500 & $\mathrm{~F}$ & 19 & 29 & 2.5 & 1600 & 0.035 & 1.9 & 0.24 & 2400 & 0.25 \\
\hline 1.1 & 5900 & $\mathrm{~F}$ & 16 & 6.0 & 1.8 & 1100 & 0.053 & 3.3 & 0.56 & 440 & 0.30 \\
\hline 1.1 & 5900 & $\mathrm{~F}$ & 16 & 6.0 & 2.0 & 1100 & 0.045 & 2.8 & 0.48 & 620 & 0.34 \\
\hline
\end{tabular}




\section{Acknowledgments}

The authors acknowledge the financial support of the University of Liège, the Faculty of Applied Science and Centre Spatial de Liège. Part of this work has been carried out within the framework of the National Centre for Competence in Research PlanetS supported by the Swiss National Science Foundation (SNSF). S. P. Q. acknowledges the financial support of the SNSF. The research leading to these results has received funding from the European Union's Horizon 2020 research and Innovation Program under Grant Agreement No. 730890 (OPTICON).

\section{References}

1. M. Mayor and D. Queloz, "A Jupiter-mass companion to a solar-type star," Nature 378, 355-359 (1995).

2. NASA, Exoplanet exploration, https://exoplanets.nasa.gov (accessed 6 August 2020).

3. G. D. Mulders, "Planet populations as a function of stellar properties," in Handbook of Exoplanets, H. J. Deeg and J. A. Belmonte, Eds., pp. 2009-2034, Springer International Publishing, Cham (2018).

4. D. J. des Marais et al., "Remote sensing of planetary properties and biosignatures on extrasolar terrestrial planets," Astrobiology 2(2) (2002).

5. C. Beichman et al., "Comparative planetology and the search for life beyond the solar system," Protostars Planets 5, 915-928 (2007).

6. R. N. Bracewell, "Detecting nonsolar planets by spinning infrared interferometer," Nature 274(5673), 780 (1978).

7. P. M. Hinz et al., "Imaging extra-solar systems from the ground: the MMT and LBT nulling interferometers," in Working on the Fringe: Opt. and IR Interferometry from Ground and Space Vol. 194, p. 401 (1999).

8. D. Defrère et al., "Exoplanet science with the LBTI: instrument status and plans," Proc. SPIE 9605, 96051G.

9. B. Mennesson et al., "Contraining the exozodiacal luminosity function of main-sequence stars: complet results from the Keck Nuller mid-infrared surveys," Astrophys. J. 797, 119 (2014).

10. D. Defrère et al., "Space-based infrared interferometry to study exoplanetary atmospheres," Exp. Astron. 46, 543-560 (2018).

11. D. Defrère, O. Absil, and C. A. Beichman, "Interferometric space missions for exoplanet science: legacy of Darwin/TPF," in Handbook of Exoplanets, H. J. Deeg and J. A. Belmonte, Eds., pp. 1229-1255, Springer International Publishing, Cham (2018).

12. S. A. Rinehart et al., "The Balloon Experimental Twin Telescope for infrared interferometry (BETTII): first flight," Proc. SPIE 10700, 107000F (2018).

13. C. B. Mendillo et al., "PICTURE: a sounding rocket experiment for direct imaging of an extrasolar planetary environment," Proc. SPIE 8442, 84420E (2012).

14. E. S. Douglas et al., "Wavefront sensing in space: flight demonstration II of the PICTURE sounding rocket payload," J. Astron. Telesc. Instrum. Syst. 4(1), 019003 (2018).

15. S. P. Quanz et al., "Exoplanet science with a space-based mid-infrared nulling interferometer," Proc. SPIE 10701, 107011I (2018).

16. J. Kammerer and S. P. Quanz, "Simulating the exoplanet yield of a space-based mid-infrared interferometer based on Kepler statistics," Astron. Astrophys. 609, A4 (2018).

17. D. Defrère et al., "Nulling interferometry: impact of exozodiacal clouds on the performance of future life-finding space missions," Astron. Astrophys. 509, A9 (2010).

18. E. Kulu, "Nanosats database," www.nanosats.eu (accessed 6 August 2020).

19. M. Nowak et al., "Reaching sub-milimag photometric precision on Beta Pictoris with a nanosat: the PicSat mission," Proc. SPIE 9904, 99044L (2016).

20. M. Nowak et al., "Short life and abrupt death of PicSat, a small 3U CubeSat dreaming of exoplanet detection,” Proc. SPIE 10698, 1069821 (2018).

21. C. Pong, "On-orbit results and lessons learned from the ASTERIA space telescope mission," https://digitalcommons.usu.edu/smallsat/2018/all2018/255/ (2018).

22. M. Knapp et al., "Demonstrating high-precision Photometry with a CubeSat ASTERIA Observations of 55 Cancri e," Astron. J. 160(1), 23 (2020). 
23. California Polytechnic State University, CubeSat, https://www.cubesat.org (accessed 6 August 2020).

24. Nanoracks, Technical Resources, https://nanoracks.com/resources/ (accessed 6 August 2020).

25. M. Casti et al., "PROBA-3 formation-flying metrology: algorithms for the shadow position sensor system," Proc. SPIE 11180, 1118082 (2019).

26. W. C. Danchi et al., "The Fourier-Kelvin Stellar Interferometer (FKSI): a review, progress report, and update," Proc. SPIE 7013, 70132Q (2008).

27. D. Defrère et al., "Nulling interferometry: performance comparison between space and ground-based sites for exozodiacal disc detection," Astron. Astrophys. 490, 435-445 (2008).

28. D. Defrère et al., "Potential of space-based infrared Bracewell interferometers for planet detection," Proc. SPIE 6693, 66931N (2007).

29. R. A. Brown, "Obscurational completeness," Astrophys. J. 607(2), 1003-1013 (2004).

30. L. Marion, "Caractérisation de disques de poussière exozodiacale par interférométrie stellaire en infrarouge proche et moyen," PhD thesis, University of Liege (2017).

31. T. Kelsall et al., "The COBE diffuse infrared background experiment search for the cosmic infrared background. II. Model of the interplanetary dust cloud," Astrophys. J. 508(1), 44-73 (1998).

32. A. Roberge et al., "The exozodiacal dust problem for direct observations of exo-Earths," Publ. Astron. Soc. Pac. 124, 799-808 (2012).

33. S. Ertel et al., "The HOSTS survey-exozodiacal dust measurements for 30 stars," Astron. J. 155, 194 (2018).

34. E. Serabyn et al., "Fiber-based interferometry and imaging," Proc. SPIE 8146, 81460I (2011).

35. B. Mennesson, M. Ollivier, and C. Ruilier, "Use of single-mode waveguides to correct the optical defects of a nulling interferometer," J. Opt. Soc. Am. A 19(3), 596 (2002).

36. S. Shaklan and F. Roddier, "Coupling starlight into single-mode fiber optics," Appl. Opt. 27(11), 2334-8 (1988).

37. C. Ruilier, "A study of degraded light coupling into single-mode fibers," Proc. SPIE 3350, 319-329 (1998).

38. G. Rieke, "Infrared detector arrays for astronomy," Annu. Rev. Astron. Astrophys. 45, 77-115 (2007).

39. E. Serabyn, "Nulling interferometry: symmetry requirements and experimental results," Proc. SPIE 4006, 328 (2000).

40. E. Serabyn et al., "Nulling at short wavelengths: theoretical performance constraints and a demonstration of faint companion detection inside the diffraction limit with a rotatingbaseline interferometer," Mon. Not. R. Astron. Soc. 489, 1291-1303 (2019).

41. O. P. Lay, "Imaging properties of rotating nulling interferometers," Appl. Opt. 44(28), 5859-71 (2005).

42. W. J. Borucki et al., "Kepler planet-detection mission: introduction and first results," Science 327(5968), 977-980 (2010).

43. NASA, Exoplanet Program: Study analysis groups, https://exoplanets.nasa.gov/exep/ exopag/sag/ (accessed 6 August 2020).

44. S. P. Quanz et al., "Direct detection of exoplanets in the 3-10 $\mu \mathrm{m}$ range with E-ELT/ METIS," Int. J. Astrobiol. 14(02), 279-289 (2015).

45. R. K. Kopparapu et al., "Exoplanet classification and yield estimates for direct imaging missions," Astrophys. J. 856(2), 122 (2018).

46. W. C. Danchi et al., "Infrared detection and characterization of debris disks, exozodiacal dust, and exoplanets: the FKSI mission concept," Pathways Towards Habitable Planets, V. Coudé du Foresto, D. M. Gelino, and I. Ribas, Eds., pp. 188-194, Astronomical Society of the Pacific, California (2010).

Colin Dandumont received his MS degrees in aerospace engineering from the University of Liège in 2018. He is a PhD student at the Centre Spatial de Liège of the University of Liège. His current research interests include interferometry, nulling interferometry, exoplanetology, and CubeSats.

Biographies of the other authors are not available. 